Z. Klin. Chem. Klin. Biochem.

13. Jg. 1975, S. 197-201

\title{
Fluoridbestimmung in Knochenproben vom menschlichen Beckenkamm mit einer ionenselektiven Elektrode
}

\author{
Von A. Zober und B. Schellmann ${ }^{1}$ ) \\ Aus dem Institut für Rechtsmedizin (Direktor: Prof. Dr. H.-B. Wu erm eling) der Universität Erlangen-Nürnberg \\ und dem \\ Pathologischen Institut (Direktor: Prof. Dr. V. Becker) der Universität Erlangen-Nürnberg
}

(Eingegangen am 9. Dezember 1974/24. Februar 1975)

Es wird eine einfach durchzuführende potentiometrische Bestimmung von anorganischem Fluorid im menschlichen Knochen mittels einer ionenselektiven Elektrode beschrieben. Die Knochenproben entstammen eincr definierten Stelle des Beckenkammes. Die Fluoridkonzentration wird aus dem Knochen nach trockener Veraschung bei $550^{\circ} \mathrm{C}$, Lösung der Asche in $\mathrm{HNO}_{3}$, $(4 \mathrm{~mol} / \mathrm{l}) \mathrm{pH}$-Einstellung mit $\mathrm{NaOH}$ ( $4 \mathrm{~mol} / \mathrm{l})$ und Pufferung mit Total Ion Strength Adjustment Buffer bestimmt. Die Auswertung erfolgt anhand einer Eichkurve, die mit wäßrigen Lösungen von NaF und Puffer gewonnen wird. Die Eichkurve ist linear im Bereich von $5,2 \mathrm{mmol} \mathrm{F}-11$ bis $10 \mu \mathrm{mol}$ $\mathrm{F}^{-} / 1$ Lösung.

Die Richtigkeit der Methode beträgt $96,1 \%$ bzw. 92,3\%. Die Präzision in der Serie, ausgedrückt durch den Pearson'schen Variabilitätskoeffizienten, liegt bei $1,68 \%$.

Im menschlichen Knochen (Beckenkamm) bestimmten wir ein Massenverhältnis zwischen 69 und $1740 \cdot 10^{-6} \mathrm{~F}^{-} / \mathrm{Asche}^{\mathrm{s}}$.

\section{Determination of fluoride in bone samples from human iliac crest with an ion-specific electrode}

A simple potentiometric method for the determination of fluoride ion in human bones is described.

The bone samples were taken from a definite position in the iliac crest.

The samples are prepared for analysis by ashing at $550^{\circ} \mathrm{C}$, dissolving in $\mathrm{HNO}_{3}(4 \mathrm{~mol} / \mathrm{l})$ and adjusting the $\mathrm{pH}$ with $\mathrm{NaOH}(4 \mathrm{~mol} / \mathrm{l})$ and Total Ion Strength Adjustment Buffer. Absolute values are derived from a calibration curve based on aqueous $\mathrm{NaF}$-buffer solutions. The calibration curve is linear in the range $5.2 \cdot 10^{-3}-1.0 \cdot 10^{-5} \mathrm{~mol} / 1 \mathrm{~F}^{-}$.

The minimum limit of measurement is $5.2 \times 10^{-6} \mathrm{~mol} / 1 \mathrm{~F}^{-}$. The method has an accuracy of $96.1 \%$ or $92.3 \%$ with a series precision based on the Pearson variability coefficient of $1.68 \%$.

The normal mass fraction of fluoride in human bones was found to be between 69 and $1740 \cdot 10^{-6} \mathrm{~F}^{-} / \mathrm{ash}$.

Erhöhte Fluoridkonzentrationen im Körper rufen toxische Wirkungen hervor und führen bei längerer Exposition zu tiefgreifenden Veränderungen der. Knochenstruktur im Sinne von Osteosklerose, Osteoporose und Mischformen dieser Osteopathien $(1,2,3)$.

Die renale Ausscheidung des Fluorids ist begrenzt, so daß - insbesondere bei Dauerexposition - Fluorid weitgehend in den Knochen abgelagert wird (4).

Die sichere und quantitative Aussage über eine Fluoridexposition ist nur durch die Kombination von histologischer und mikrochemischer Untersuchung von bioptisch gewonnenem Knochenmaterial aus dem Beckenkamm der betroffenen Personen möglich (5).

Die Fluoridbestimmung in Knochenproben wurde bisher mit einer Vielzahl analytischer Methoden durchgeführt

1) Bei der Deutschen Forschungsgemeinschaft bedanken wir uns für die Bereitstellung von Sachmitteln.
(6-13). Beim Vergleich der so gewonnenen Werte ist den unterschiedlichen Techniken Rechnung zu tragen. Die Fluoranalytik wurde nach Entwicklung der ionenspezifischen Fluoridelektrode durch Frant \& Ross (1966) erheblich erleichtert, da bisher notwendige zeitraubende Destillations- und Diffusionsverfahren entfallen können. Die Messungen des Fluorids mit der Elektrode in Knochen von Ratten sowie in Fischgräten war Gegenstand dreier Publikationen in den Jahren 1968 bis 1970 (14-16).

Messungen des Fluoridgehaltes von menschlichen Knochen unter Verwendung der ionenspezifischen Fluoridelektrode liegen bisher nicht vor. Ältere Arbeiten mit anderen Methoden beziehen sich hauptsächlich auf Verhältnisse in den USA. Die dort gefundenen Werte sind nicht ohne weiteres auf hiesige Verhältnisse zu übertragen, da der Fluorgehalt im Knochen u. a. mit dem Fluoridgehalt des über längere Zeit aufgenommenen Trinkwassers korreliert. Dadurch sind regionale 
Unterschiede bei der physiologischen Fluoridkonzentration im Knochen zu erwarten.

Es wird in der folgenden Arbeit eine geeignete Methodik für die Fluoridbestimmung in menschlichen Knochenproben angegeben. Kriterien für die Zuverlässigkeit sind Präzision und Richtigkeit. Das Vorgehen bei der Probenahme wird standardisiert analog der klinischen perkutanen Punktionstechnik (17), um im Hinblick auf Gutachtensproblematik immer vergleichbare Werte zu erhalten. Die Entnahme der Knochenproben erfolgte an Leichen beiderlei Geschlechts aus hiesigem Sektionsgut im Alter von 6 Monaten bis 97 Jahren.

\section{Methodik und Material \\ Prinzip der Meßmethode}

Es handelt sich um eine potentiometrische Bestimmung von anorganischem Fluorid mittels einer ionenselektiven Elektrode aus menschlichen Knochenproben nach Veraschung. Die Messung der Fluoridkonzentration erfolgt bei definiertem $\mathrm{pH}$, konstanter Ionenstärke und Anwesenheit von Substanzen, die stabile Fluorkomplexe dekomplexieren können (TISAB = Total Ion Strength Adjustment Buffer).

Eine gesättigte Kalomelelektrode dient als Bezugselektrode. Als Eichlösungen finden wäßrige Natriumfluoridlösungen Verwendung, die in gleicher Weise wie die in Lösung gebrachte Knochenasche behandelt werden. Nachweisgrenze: $5,2 \mu \mathrm{mol}$ $\mathrm{F}^{-} / 1$ Lösung.

Technik der Methode

Probenahme

Das Knochenmaterial wurde $3 \mathrm{~cm}$ dorsal der rechten Spina iliaca anterior superior durch Sägeschnitt in $5 \mathrm{~mm}$ starken, etwa $3 \mathrm{~g}$ schweren Proben entnommen. Nach Entfernung des Muskel- und Fasciengewebes wird das Frischgewicht auf $0,01 \mathrm{~g}$ genau festgelegt. Zwischen Entnahme und Wägung wird die Probe in einer feuchten Kammer aufbewahrt. Daran schließt sich eine Entwässerung im Vakuumtrockenschrank bei 200 Torr und $90^{\circ} \mathrm{C}$ über 4 Stunden an.

\section{Veraschung}

Die getrockneten Knochenproben werden im Muffelofen ohne Zusätze unter Luftzutritt bei $550^{\circ} \mathrm{C}$ während 15 Stunden im bedeckten Platintiegel verascht. Der Veraschungsrückstand wird im Achatmörser fein verrieben und aliquote Anteile auf der Analy senwaage zur Messung eingewogen.

\section{Geräte, Lösungen}

\section{Geräte}

Millivoltmeter mit gespreizter Skala bzw. digital anzeigendes Millivoltmeter mit gespreizter Skala (Ablesegenauigkeit 0,1 mV). Fluoridspezifische Elektrode (z. B. ORION)

gesättigte Kalomel-Referenzelektrode

Glaselektrode

Muffelofen

Magnetrührer mit Rührstab (Teflon).

\section{Lösungen}

Alle verwendeten Lösungen sollen stets in Polyäthylen-Schraubgefäßen aufbewahrt werden.

\section{Standardlösungen}

Die wäßrige Stammlösung von $52 \mathrm{mmol} \mathrm{F}^{-} / 1$ ist bei Kühlung unbegrenzt haltbar. Aus der Stammlösung werden - wie bei Sunderman (18) beschrieben - die entsprechenden Verdünnungen von 5,$2 ; 2,6 ; 1,04 \mathrm{mmol} / 1$ usw. bis $5,2 \mu \mathrm{mol} \mathrm{F}-11$ hergestellt Die Standardlösung Nr. I von $5,2 \mathrm{mmol} \mathrm{F}^{-} / 1$ ist im Kühlschrank eine Woche haltbar. Alle weiteren Standardlösungen sollen jeweils frisch bereitet werden.
Puffer (Total Ion Strength Adjustment Buffer $=$ TISAB). Dieser Puffer kann nach den Angaben von Frant \& Ross (19) selbst hergestellt oder auch im Handel-(ORION) erworben werden.

\section{Kalibrierung}

Vor der Kalibirierung wird die $\mathbf{F}^{-}$-spezifische Elektrode einige Stunden in der Standardlösung Nr. I konditioniert. AnschlieBend taucht man die Elektrode einige Sekunden in deionisiertes Wasser und trocknet die Membran mit weichem Papier ab.

Zur Kalibrierung pipettiert man $5 \mathrm{ml}$ Puffer ynd $5 \mathrm{ml}$ Standard Nr. I in das Polyäthylen-Schälchen, taucht Fluorid- und Kalomelvergleichs-Elektrode bis kurz unter die Flüssigkeitsoberflächliche und rührt 10 Minuten. Nun wird zu diesem 5,2 mmol

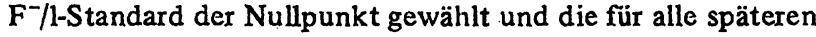
Messungen verbindliche Arbeitstemperatur festgestellt. Nach weiteren 30 Minuten wird der Nullpunkt - bei dauerndem Rühren und Temperaturkontrolle - wenn nötig, nachgeeicht.

Anschließend werden in 3 fach-Messungen, von der größten Verdünnung her beginnend, alle Standardkonzentrationen gemessen. Nach 2-8 Stunden muß eine Kontrollmessung des Nullpunktes mit dem Standard Nr. I in die Meßrcihe eingeführt werden. Die Einstellungszeit des mV-Meters zur Konstanz ist kürzer, wenn von verdünnten $z u$ konzentrierten Lösungen übergegangen wird. Die Mittelwerte werden auf der linearen Abszisse eines DIN A 3 halblogarithmischen Papiers abgetragen, die zugehörigen $\mathbf{F}^{-}$-Konzentrationen auf der logarithmischen Ordinate (Nernst-Gleichung). Die Steigung soll bei $22^{\circ} \mathrm{C}$ etwa $58 \mathrm{mV}$ pro Dekade betragen. Zwischen 5,2 $\mathrm{mmol} \mathrm{F}^{-} / 1$ und $10 \mu \mathrm{mol}$ $\mathrm{F}^{-} / 1$ ist die Kurve linear, denn treten geringe $\mathrm{Abweichungen}$ auf (je nach verwendeter Elektrode). Eine Eichkurve findet sich in Abbildung 1.

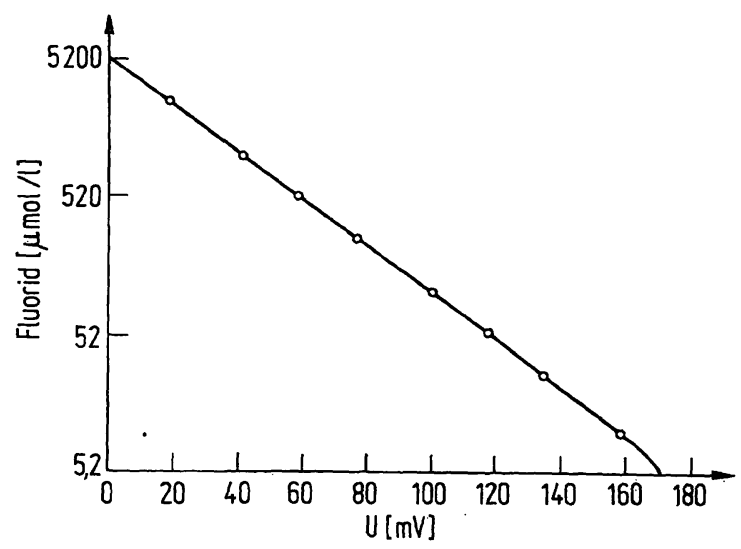

Abb. 1. Eichkurve zur Fluorid-Bestimmung mit wäßriger NaFLösung und Total Ion Strength Adjustment Buffer bei $\mathrm{T}=22^{\circ} \mathrm{C}$.

\section{Analytische Bestimmung}

Nach Möglichkeit werden etwa $100 \mathrm{mg}$ pulverisierter Knochenasche ausgewogen und in einen Polyäthylen-Becher überführt. Die Asche wird durch Zutropfen von $2 \mathrm{ml} \mathrm{HNO}_{3}(4 \mathrm{~mol} / \mathrm{l})$ während 3 bis 5 Minuten gelöst. Diese Lösung wird mit deionisiertem Wasser in einen $25 \mathrm{ml}$ Meßkolben gespült und $1 \mathrm{ml}$ $\mathrm{NaOH}(4 \mathrm{~mol} / \mathrm{l})$ zugetropft. Den sich bildenden flockigen Niederschlag bringt man durch Schütteln wieder in Lösung. Nun füllt man mit deionisiertem Wasser zur Marke auf. $5 \mathrm{ml}$ dieser Probe und $5 \mathrm{ml}$ Puffer werden in einen Polyäthylen-Meßbecher pipettiert. Es resultiert ein pH-Wert zwischen 5 und 5,5.

Die Elektroden werden bis knapp unter die Flüssigkeitsoberfläche eingeführt. Nun wird bis zur Konstanz der Anzeige gerührt, die im allgemeinen innerhalb 5-10 Minuten eintritt.

Dabei muß das Polyäthylen-Gefäß auf einer Asbestplatte stehen, um Temperatureffekte durch den Rührmotor zu vermeiden. 
Reinigung der Elcktroden nach jeder Messung:

Kurzes Eintauchen in deionisiertes Wasser, Abtrocknen mit weichem Papicr.

Von Zeit zu Zeit sollte eine Messung mit $5 \mathrm{ml}$ Puffer und $5 \mathrm{ml}$ $\mathrm{HNO}_{3} / \mathrm{NaOH}$ eingeschoben werden, um eine Fluoridf reiheit der verwendeten Lösungen zu gewährleisten. Die Anzeige muß dann außerhalb der gewählten Eichkurve liegen (hicr größer $180 \mathrm{mV}$ ). Bei größeren Aschenmengen muß die $\mathrm{HNO}_{3} / \mathrm{NaOH}-$ Zugabe variiert werden. Es gilt beispielsweise für:

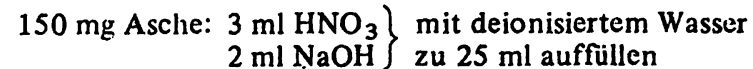

$1000 \mathrm{mg}$ Asche: $8 \mathrm{ml} \mathrm{HNO}_{3}$ mit deionisiertem Wasser $5 \mathrm{ml} \mathrm{NaOH}\}$ zu $50 \mathrm{ml}$ auffüllen

Bei kleineren Aschenmengen als $100 \mathrm{mg}$ muß das Verhältnis $\mathrm{HNO}_{3} / \mathrm{NaOH}$ sinngemäß geändert werden.

\section{Berechnung der Ergebnisse}

Dic Ablesung erfolgt direkt in $\mu \mathrm{mol} \mathrm{F}-/ 1$ Lösung. Der Fluoridgehalt der eingewogenen Aschenmenge ergibt sich nach Multiplikation mit 25 (oder dem entsprechenden Lösungsvolumen).

Die endgültige Angabe soll im Massenverhältnis Fluorid/Asche $\times 10^{-6}$ erfolgen.

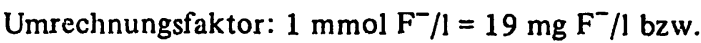

$52 \mu \mathrm{mol} \mathrm{F}^{-} / 1=1 \mathrm{mg} \mathrm{F}^{-} / 1$.

\section{Kontrolle des Verfahrens}

\section{Präzision}

Zur Bestimmung der Präzision in der Serie wurden 10 mal stets neu ausgewogene Ascheanteile von etwa 100 mg einer Knochenprobe analysiert.

Aus den Ergebnissen folgt für ein mittleres Massenverhältnis von $640,3 \times 10^{-6} \mathrm{~F}^{-} /$Asche eine Standardabweichung von $10,78 \times 10^{-6} \mathrm{~F}^{-} /$Asche.

Der Pearson'sche Variabilitätskoeffizient beträgt 1,68\%. Dic Messungen erfolgten während 8 Stunden, in denen der Nullpunkt des $\mathrm{mV}$-Meters nicht nachgeeicht wurde.

\section{Richtigkeit}

Zur Festlegung der Richtigkeit wurden Zusatzversuche durchgeführt. Wie beschrieben entnimmt man aus dem Beckenkamm eine Knochenprobe I zur Bestimmung des Fluoridnormalwertes.
Durch Sägeschnitt direkt ncben der Entnahmestelle I wird ein weiteres Knochenstück II für die Zusatzversuche gewonnen. Der Kompakta- und Spongiosaanteil der Proben I und II muß gleich sein, um einen identischen Fluorgehalt beider Knochenstücke zu gewährleisten. Der Fluoridgehalt von Probe I wird wic angegeben bestimmt. Zur getrockneten Probe II fügt man im Platintiegel 500 bzw. $750 \mu \mathrm{g}$ Fluorid in Form wäßriger Natriumfluoridlösung durch Eintropfen in die Spongiosa zu. Nach erneuter 24 stündiger Trocknung wird die so präparicrte Probe II verascht und der Fluoridgehalt der Gesamtaschemenge bestimmt. Die Berechnung zur Richtigkeit erfolgte unter Bezug auf den Fluorwert von Probe I. Da die Aschemenge beim Zusatzversuch etwa $800-1000 \mathrm{mg}$ beträgt, sind $8 \mathrm{ml} \mathrm{HNO}{ }_{3}(4 \mathrm{~mol} / \mathrm{l})$ zur Lösung crforderlich. Die Asche wird direkt im Platintiegel zur Lösung gebracht. Das Gesamtvolumen überführt man in einen $50 \mathrm{ml}$ Meßkolben, wo zur pH-Einstellung 4-5 $\mathrm{ml} \mathrm{NaOH} \mathrm{(4} \mathrm{mol/l)} \mathrm{zu-}$ getropft werden. Zur Messung muß erncut verdünnt werden. Man entnimmt $5 \mathrm{ml}$ aus dem zur Marke aufgefüllten $50 \mathrm{ml}$ Meßkolben und füllt zu $25 \mathrm{ml}$ mit deionisiertem Wasser im 2. Mcßkolben auf. Erst von dieser Verdünnung werden $5 \mathrm{ml}-$ Proben mit $5 \mathrm{ml}$ Puffer vermischt und der Fluoridgehalt bestimmt. Die Verdünnungsschritte sind bei der Berechnung zu berücksichtigen. Die Richtigkeit liegt bei Zugabe von $500 \mu \mathrm{g}$ $F^{-}$im Mittel bei $96,1 \%$, bei Zugabe von $750 \mu \mathrm{g} \mathrm{F}$ bei $92,3 \%$, (siehc Tab. 1).

\section{Nachweisgrenze}

Unter den angegebenen Bedingungen beträgt dic Nachwcisgrenze $5,2 \mu \mathrm{mol} \mathrm{F}-11$ Lösung. Die theoretische Nachweisgrenze liegt mit $1,0 \mu \mathrm{mol} \mathrm{F}^{-} / 1 \mathrm{im}$ Gebiet der $\mathrm{F}^{-}$-Konzentrationen, die durch das Löslichkeitsprodukt der Lanthanfluorid-Membran in Lösung gebracht werden. Die Bestimmung der praktischen Nachwcisgrenze über den Störpegel (bzw. den Leerwert) ist bei Messungen mit dem mV-Meter nicht sinnvoll. Als Nachweisgrenze wird deshalb die Stoffmengen-Konzentration an Fluorid angenommen, bis zu der die Eichkurve bei allen geprüften Elektroden linear verläuft, bei größeren Verdünnungen als $5,2 \mu \mathrm{mol} \mathrm{F} / /$ weicht die Eichkurve stark und nicht reproduzierbar von der Linearität ab.

\section{Ergebnisse}

Mit der angegebenen Methodik wurden 100 Knochenproben von 100 Leichen beiderlei Geschlechts analysiert. Die Werte lagen als Massenverhältnis zwischen 69 und $1740 \times 10^{-6} \mathrm{~F}^{-} /$Asche.

Tab. 1. Meßwerte zur Richtigkeit der Methode

A: Probe 1-5 Richtigkeit bei Zugabe von $500 \mu \mathrm{g} \mathrm{F}^{-}$zur Knochenprobe

B: Probe 6-10 Richtigkeit bei Zugabe von $750 \mu \mathrm{g} \mathrm{F}^{-}$zur Knochenprobe

\begin{tabular}{|c|c|c|c|c|c|c|}
\hline Probe & $\begin{array}{l}\text { Normalwert } \\
\text { Massenverhältnis } \\
\mathrm{F}^{-} / \text {Ascheeinwaage } \\
\text { für Probe II } \\
\times 10^{-6} \\
\text { (berechnet aus } \\
\text { Probe I) }\end{array}$ & $\begin{array}{l}\text { Ascheeinwaage } \\
\text { Probe II } \\
\text { in } \mathrm{mg}\end{array}$ & $\begin{array}{l}\text { Zusatz } \mu \mathrm{g} \mathrm{F}^{-} \\
\text {zu Probe II }\end{array}$ & $\begin{array}{l}\text { gefunden } \\
\text { Massenverhältnis } \\
\mathrm{F}^{-} / \text {Gesamt- } \\
\text { einwaage } \\
\times 10^{-6} \\
\text { Probe II }\end{array}$ & $\begin{array}{l}\text { wiedergefunden } \\
\mu \mathrm{g} \mathrm{F}^{-} \\
\text {in Probe II }\end{array}$ & $\begin{array}{l}\text { Wieder- } \\
\text { auffindung } \\
\text { in } \%\end{array}$ \\
\hline $\begin{array}{r}1 \\
2 \\
3 \\
4 \\
5\end{array}$ & $\begin{array}{l}72,9 \\
104,9 \\
177,9 \\
197,7 \\
248,6\end{array}$ & $\begin{array}{l}416,8 \\
788,6 \\
407,2 \\
255,8 \\
812,5\end{array}$ & $\begin{array}{l}500 \\
500 \\
500 \\
500 \\
500\end{array}$ & $\begin{array}{l}525 \\
575 \\
700 \\
675 \\
725\end{array}$ & $\begin{array}{l}452,1 \\
470,1 \\
522,0 \\
477,3 \\
476,4\end{array}$ & $\begin{array}{r}90,4 \\
94,0 \\
104,4 \\
96,9 \\
95,0 \\
96,1 \\
==\end{array}$ \\
\hline $\begin{array}{r}6 \\
\text { B } \quad 8 \\
9 \\
9 \\
10\end{array}$ & $\begin{array}{r}66,5 \\
120,3 \\
144,3 \\
405,4 \\
477,1\end{array}$ & $\begin{array}{l}635,8 \\
421,2 \\
767,7 \\
791,0 \\
580,2\end{array}$ & $\begin{array}{l}750 \\
750 \\
750 \\
750 \\
750\end{array}$ & $\begin{array}{r}725 \\
800 \\
900 \\
1100 \\
1150\end{array}$ & $\begin{array}{l}658,5 \\
679,7 \\
755,7 \\
694,5 \\
672,9\end{array}$ & $\begin{array}{r}87,8 \\
90,9 \\
100,6 \\
92,6 \\
89,7 \\
92,3 \\
===\end{array}$ \\
\hline
\end{tabular}




\section{Diskussion}

Die hier beschriebene Methode der potentiometrischen Fluoridbestimmung im Knochen wird erstmals an menschlichem Material durchgeführt und durch Angabe von Präzision und Richtigkeit kritisch bewertet.

Im Gegensatz zu den Untersuchungen von Barnes \& Runcie (14) haben wir beim Veraschungsvorgang keine Zusätze in Form von Lithiumhydroxid bzw. Magnesiumsuccinat zur etwaigen Bindung flüchtigen Fluorides verwendet, da die trockene Veraschung im basischen Milieu stattfindet; wäßrige Auszüge der pulverisierten Knochenasche zeigten $\mathrm{pH}$-Werte zwischen 8,5 und 11,5.

Damit ist der Verlust von Fluorid in Form gasförmiger Flußsäure auszuschließen.

Die bei der Veraschungstemperatur von $550{ }^{\circ} \mathrm{C}$ im Knochen vorliegenden Fluorapatite sind sehr stabil und haben einen weit höheren Verdampfungspunkt.

Die verwendeten Tiegel müssen einen Deckel besitzen, um Kontamination aus dem Schamott des Muffelofens zu vermeiden. Bei Durchfuhrung der Wiederauffindungsversuche zur Festlegung der Richtigkeit sollen Platintiegel verwendet werden, da eine Lösung der Gesamtasche zur Erfassung der Zusätze direkt im Tiegel mit Salpetersäure stattfinden muß; bei Routineanalysen erwiesen sich Nickeltiegel als gleichwertig.

Ein Aufschluß der Asche mit $\mathrm{NaOH}$ - wie bei Oelschläger \& Wöhlbier (21) angegeben - ist nicht erforderlich. Unsere vergleichenden Untersuchungen ergaben bei direkter Lösung der Asche in Salpetersäure gleiche Werte wie nach alkalischem Aufschluß.

Zur Lösung der Asche menschlichen Knochens wurde von uns - wie bei $\mathrm{Ke}$ et al. (16) - $\mathrm{HNO}_{3}(4 \mathrm{~mol} / \mathrm{l})$ ver-

\section{Literatur}

1. Weidmann, S. M., Weatherell, J. A. \& Jackson, D. (1963), Proc. Nutr. Soc. 22, 105-110.

2. Minder, W., in Toxikologie des Fluors (1964) (Gordonoff, T. ed.) pp. 80-90 Schwabe \& Co. Verlag Basel/Stuttgart.

3. Krishnamachari, K. A. V. R. \& Krishnaswamy, K. (1973), Lancet $I I, 877-879$.

4. Weatherell, J. A. (1966), in Heffter, Heubner, Handbuch der experimentellen Pharmakologie, Pharmacology of Fluorides Part. 1. (Eichler, O., Farah, A., Herken, H., Welch, A. D., ed.) pp. 141-172, Springer Verlag, Berlin, Heidelberg, New York.

5. Franke, J. \& Auermann, E. (1972), Int. Arch. Arbeitsmed. 29, 85-94.

6. Bäumler, J. \& Glinz, E. (1964), Mitt. Lebensm. Untsg. Hyg. 55, 250-264.

7. McHargue, J. C. \& Hodkins, W. S. (1939), J. Ass. Offic. Agric. Chem. 22, 249-254. wendet, da es fraglich erscheint, ob $\mathrm{HCl}(0,25 \mathrm{~mol} / \mathrm{l})-$ die Singer \& Armstrong (15) angeben - ausreichende Lösung der nach Veraschung vorliegenden Fluorapatite herbeiführt; bei uns waren Lösungsversuche mit $\mathrm{CaF}_{2}$ und $\mathrm{HCl}(0,25 \mathrm{~mol} / \mathrm{l})$ unbefriedigend. In diesem Zusammenhang überprüften wir auch die Löslichkeitsverhältnisse bei der Aufstellung der Eichkurve. Dabei ergaben sich identische Kurven für wäßrige NaF- und salpetersaure $\mathrm{CaF}_{2}$-Lösungen.

Für die Messung selbst sind bezüglich der Elektrode verschiedene Störeinflüsse möglich.

Von Bedeutung ist der $\mathrm{pH}-$ Bereich der Meßlösung, der unbedingt zwischen 5 und 7 (vor allem bei geringen $\mathrm{F}^{-}$-Konzentrationen) liegen muß (22).

Die Potentialanzeige wird inkonstant bei gealterten Elektroden (etwa 2 Jahre) oder zerstörten Membranen (Rührer!). Falsch positive Werte treten bei eingeschlepptem Fluorid aus Chemikalien oder aus dem deionișierten Wasser auf (Blindmessungen!). Temperaturunterschiede sowie ungenügende Nullpunktnacheichung bedingen schwankende Meßwerte. Unterschiede von $2{ }^{\circ} \mathrm{C}$ entsprechen dabei $0,5 \mathrm{mV}$.

Bei der Bestimmung von Fluoridnormalwerten im Knochen werden primäre oder renale Osteopathien aus dem Untersuchungsgut ausgeschlossen.

Im Hinblick auf gute Vergleichbarkeit von Normalwerten und Punktaten ist auf annähernd konstantes Kompakta/Spongiosa-Verhältnis zu achten. Singer \& Armstrong (23) haben darauf hingewiesen, daß spongiöser Knochen etwa 1,5-3 mal mehr Fluorid enthält als kompakter Knochen.

8. Danckwortt, P. W. (1941), Hoppe-Seyler's Z. Physiol. Chem. 268, 187-193.

9. Cremer, H. D. \& Voelker, W. (1953), Biochem. Z. 324, 89-92.

10. Samachson, J., Slovik, N. \& Sobel, A. E. (1957), Anal. Chem. 29, 1888-1891.

11. Jackson, D. \& Weidmann, S. M. (1958), J. Pathol. Bact. 86, $451-459$.

12. Azar, A. (1967), Ann. Intern. Med. 55, 193-200.

13. Dost, F. N., Reed, D. J., Cooper, T. B. \& Wang, C. H. (1970), Toxicol. Appl. Pharmacol. 17, 573-5.84.

14. Barnes, F. W. \& Runcie, J. (1968), J. Clin. Pathol. 21, 668-670. 15. Singer, L. \& Armstrong, W. D. (1968), Anal. Chem. 40,
613-614.

16. Ke, J., Power, H. E. \& Regier, L. W. (1970), J. Sci. Food Agr. 21, 108-109.

17. Wagner, H. (1965), Präsenile Osteoporose, pp. 36-40, G. Thieme Verlag Stuttgart. 
18. Sunderman, F. W. \& Sunderman, F. W., Jr. (1970) Laboratory Diagnosis of Diseases caused by toxic agents pp. 215-217 Warren H. Green Inc. St. Louis, Miss., USA. H. Green Inc. St. Louis, Miss., USA.

19. Frant, M. S. \& Ross, J. W. (1968), Anal. Chem. 40, 11691171.

20. Schellmann, B. \& Zober, A. (1975), Int. Arch. Arbeitsmed., im Druck.
21. Oelschläger, W. \& Wöhlbier, W. (1968), in Fluor-Wirkungen, Forschungsberichte 14 DFG, pp. 6-27, Franz Steiner Verlag Wiesbaden.

22. Cammann, K., (1973), Das Arbeiten mit ionenselektiven Elektroden, pp. 67-74. Springer Verlag Berlin, Heidelberg, New York.

23. Singer, L. \& Armstrong, W. D. (1962), J. Dent. Res. 41, $154-157$.
Dr. Andreas Zober Institut für Rechtsmedizin der Universität

Erlangen-Nürnberg

Universitätsstraßc 22 
